\title{
Introduction of Generator Unit Controller and Its Tuning for Automatic Generation Control in Korean Energy Management System (K-EMS)
}

\author{
Min-Su Park* and Yeong-Han Chun ${ }^{\dagger}$
}

\begin{abstract}
Automatic generation control (AGC) is an important function for load frequency control, which is being implemented in Energy Management System (EMS). A key feature of AGC is to back up governors to enhance the performance of frequency control. The governor regulates system frequency in several to ten seconds, while the droop control concept results in steady-state control error. AGC is a supplementary tool for compensation of the steady-state error caused by the droop setting of the governors. As the AGC target is delivered to each generator as an open loop control target, the generator output is not guaranteed to follow the AGC target. In this paper, we introduce generating unit controller (GUC) control block, which has the purpose of enabling the generator output to track the AGC target while maintaining the governor performance. We also address the tuning methods of GUC for better performance of AGC in the Korea Energy Management System (K-EMS).
\end{abstract}

Keywords: AGC, LFC, Frequency control, EMS, GUC

\section{Introduction}

The present automatic generation control (AGC) scheme has evolved over more than 70 years and has been widely used in the world[1]. Korea Power Exchange (KPX) also utilizes AGC function in the Energy Management System (EMS) installed in the control center and is now developing new EMS (K-EMS) with advanced information technology (IT). As there are several control blocks for the balancing of supply-demand mismatch[2], it is very important to differentiate each function on the time frame. Supply-load balancing can be achieved by economic dispatch (ED), load frequency control (LFC), tracking ED (TED), and generating unit control (GUC) blocks, while the governor acts as primary control by regulating the speed of each generator. GUC is a control function implemented in Korean EMS (K-EMS) to enable the generator output to follow the AGC target signal.

N. Jaleeli et al.[1] addressed the concept of AGC in comparison with the governor. The purpose of AGC is to replace portions of manual control. As it automatically responds to normal load changes, AGC reduces the response time to approximately a minute or two. The performance of $\mathrm{AGC}$ can be obtained by making the response time longer than that of the governor. In the United States, the procedure in most control areas requires the AGC to be suspended when the frequency deviates $200 \mathrm{mHz}$ or more. AGC is set to respond only to normal load changes. When

\footnotetext{
$\dagger \quad$ Corresponding Author: Department of Electrical and Electronic Engineering, Hongik University, Korea. (yhchun@hongik.ac.kr)

* Department of Electrical and Electronic Engineering, Hongik University, Korea. (mspark_yah@yahoo.co.kr)

Received: October 11, 2010; Accepted: December 13, 2010
}

changes of generation due to governor action are not enough to hold the runaway frequency, load shedding or tripping generating units are adopted by UFR or OFR to prevent system collapse.

The primary function of AGC is for LFC, which backs up the performance of the governor in multi-machine systems[3], [4]. The objective of LFC is to regulate frequency with longer response time than the response of the governor action, especially in normal system conditions. Although the governor is the fastest controller that responds to the frequency deviation, steady-state error due to droop control property remains. AGC calculates the area control error (ACE) and distribute it to each generating unit proportional to the participation factors; it then sums the ED signal and distributed ACE to generate the target signal of each generating unit. In the K-EMS, GUC logic is introduced for tracking the target signal by providing feedback to the output power through SCADA[5].

\section{AGC in K-EMS}

\subsection{Data Filtering}

Data obtained through SCADA are processed by lowpass filter to eliminate the effect of noise and random load changes.

$$
y(t)=\frac{1}{1+s T} x(t)
$$

$x(t):$ Filter input 
$y(t):$ Filter output

$T:$ Time constant

The data processed by low-pass filter are ACE and TED. The time constant of each filter differs according to the data property and control response time. The ACE time constant is set to be larger than the governor time constant to avoid conflict between the two responses. The TED time constant is set to be larger than the ACE time constant for the same reason. Two time constants set on the EMS is given in Table 1.

Table 1. Time constant table

\begin{tabular}{c|c|c}
\hline Function & ACE & TED \\
\hline Seconds & 60 & 90 \\
\hline
\end{tabular}

\subsection{ACE Calculation}

ACE consists of frequency deviation term and tie-line power deviation term, as shown in equation (2)[7], [8].

$$
\mathrm{ACE}=10 \times B \times(F S-F-T E)+\text { Ints }- \text { Int }
$$

$B:$ Frequency bias factor

$F:$ System frequency

$F S$ : Scheduled frequency

$T E$ : Time error

Ints : Summation of tie-line power flow

Int : Scheduled summation of tie-line power flow

$\mathrm{ACE}$ is distributed to each generating unit according to the regulation participation factor (RPF), which is determined by the ramp rate of each generating unit. RPF is calculated by equation (3).

$$
R P F_{i}=R R_{i} /\left(\sum_{i=1}^{n} R R_{i}\right)
$$

$R P F_{i}$ : RPF of generating unit $\mathrm{i}$

$R R_{i}$ : Ramp rate of generating unit $\mathrm{i}$

$\sum_{i=1}^{n} R R_{i}$ : Sum of ramp rates of generating units

\subsection{TED}

$\mathrm{ED}$ in the K-EMS is calculated every minute; it generates base points of generating units. LFC calculates ACE every four seconds, and ACE is distributed to the generating units proportional to the participating factors. In contrast, TED calculates TEDMW (megawatts result of TED) with the deviation between the sum of the ED base points and the system loads. TED is executed every four seconds and distributed according to the economic participation factor (EPF). TED is introduced in the AGC logic to reflect the load effects of the system.

EPF is determined by the incremental cost $\lambda$ of generating units. It is calculated differently according to the sum of the ED base points and the system loads. When the sum of the ED base points is greater than the system load, EPF is calculated proportionally to $\lambda$ and vice versa. EPF is calculated as in equation (4).

$E P F_{i}=\left(1 / \lambda_{i}\right) /\left(\sum_{i=1}^{n} 1 / \lambda_{i}\right)$ when $\sum_{i=1}^{n} B P_{i}>$ system load

$E P F_{i}=\left(\lambda_{i}\right) /\left(\sum_{i=1}^{n} \lambda_{i}\right)$ when $\sum_{i=1}^{n} B P_{i}<$ system load

\section{$E P F_{i}: \mathrm{EPF}$}

$\lambda_{i}$ : Incremental cost of generating unit $\mathrm{i}$

$B P_{i}$ : Base point of generating unit i from ED

As ACE and TED are calculated every four seconds, it is important to avoid conflicts of control action by setting response time differently. It can be accomplished by deciding LFC time constants differently. ACE is distributed according to ramp rate while TED is distributed according to the incremental costs, it is reasonable to make the TED time constant to be larger than that of ACE time constant.

\subsection{AGC Control Signal}

Final target signals from AGC are determined by the sum of the ED base points, ACE terms, and TED terms as in equation (5)[9].

$$
P t_{\mathrm{i}}=B P_{i}+A C E \times R P F_{i}+T E D M W \times E P F_{i}
$$

\section{$P t_{i}$ : Target signal from AGC to generating unit $\mathrm{i}$}

\section{GUC}

GUC is defined as the controller that has been implemented in the K-EMS to track the AGC target signals, as shown in Fig. 1. The generator power output is not guaranteed to follow the AGC target signals without GUC due to governor droop action. On the other hand, the GUC can deteriorate the governor performance if its gain is tuned to have excessively fast response.

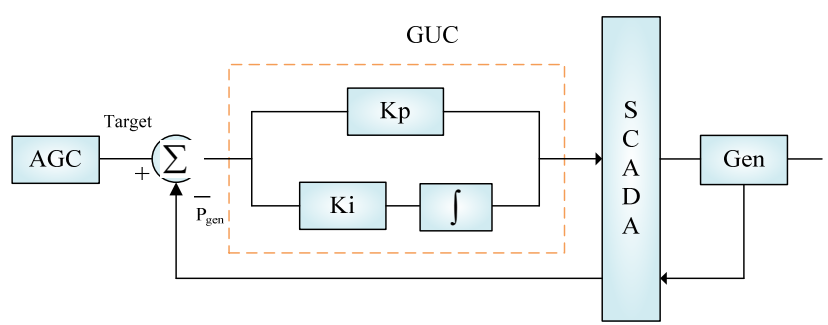

Fig. 1. Block diagram of GUC. 
Therefore, the tuning of the GUC is a trade-off between the AGC tracking performance and the governor performance. In this paper, we address a tuning method based on PI controller and model reduction. The transfer function of PI controller is shown in equation (6)[10].

$$
K(s)=K_{p}+\frac{K_{i}}{s}
$$

Where $K_{p}$ is the proportional gain and $K_{i}$ is the integral gain.

\subsection{Linearized Model and Model Reduction}

Large power systems can be simplified by a twogenerator system, one being the generator involving the GUC tuning and the other one which is the fictitious generator equivalent to the rest of the generators concerned, as shown in Fig. 2.

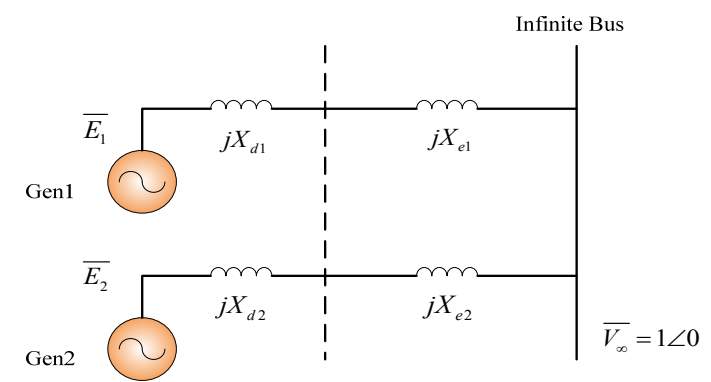

Fig. 2. Two-generator equivalent system.

\subsection{Linear Model of Generator[11]}

\section{The $E_{q}^{\prime}$ equation}

We can obtain the equation using field equation and generator terminal voltages $v_{d}$ and $v_{q}$.

$$
\begin{gathered}
E_{q \square}^{\prime}=\frac{K_{1}}{1+K_{1} \tau_{d o}^{\prime} s} E_{F D \square}-\frac{K_{1} K_{2}}{1+K_{1} \tau_{d o}^{\prime} s} \delta \\
K_{1}=1 /\left(1+K_{I}\left(x_{q}+X_{e}\right)\left(x_{q}+X_{e}\right)\right) \\
K_{2}=V_{\infty} K_{I}\left(x_{d}-x_{d}^{\prime}\right) \times \\
\quad\left[\left(x_{q}+X_{e}\right) \sin \left(\delta_{0}-\alpha\right)-R_{e} \cos \left(\delta_{0}-\alpha\right)\right]
\end{gathered}
$$

Where $K_{1}$ is the impedance factor and $K_{2}$ is related to de magnetizing effect of a change in the rotor angle.

\section{The electrical torque $T_{e}$ equation}

$$
T_{e \square} \square K_{3} \delta_{\square}+K_{4} E_{q \square}^{\prime}
$$

$$
\begin{aligned}
K_{3}= & K_{I} V_{\infty}\left\{E_{q a 0}\left[\sin \left(\delta_{0}-\alpha\right)+\left(x_{d}^{\prime}+X_{e}\right) \cos \left(\delta_{0}-\alpha\right)\right]\right. \\
+ & \left.I_{q 0}\left(x_{q}-x_{d}^{\prime}\right)\left[\left(x_{q}+X_{e}\right) \sin \left(\delta_{0}-\alpha\right)-R_{e} \cos \left(\delta_{0}-\alpha\right)\right]\right\} \\
& K_{4}=K_{I}\left\{I_{q 0}\left[R_{e}^{2}+\left(x_{q}+X_{e}\right)^{2}\right]+E_{q a 0} R_{e}\right\}
\end{aligned}
$$

Where $K_{3}$ is the change in electrical torque for small change in rotor angle at constant $\mathrm{d}$ axis flux linkage and $K_{4}$ is the change in electrical torque for small change in the $\mathrm{d}$ axis flux linkage and constant rotor angle.

$$
\begin{gathered}
\left.K_{3}=\frac{T_{e \square}}{\delta \square}\right]_{E_{q}^{\prime}=E_{q 0}^{\prime}} \\
\left.K_{4}=\frac{T_{e \square}}{E_{q \square}^{\prime}}\right]_{\delta=\delta_{0}}
\end{gathered}
$$

$K_{1}, K_{2}, K_{3}$, and $K_{4}$ depend on the network parameter, the quiescent operating condition, and the infinite bus voltage.

\section{Generator rotor angle $\delta$}

The block diagram $\delta$ is shown in Fig. 3 .

$$
M \omega_{\square}+D \omega_{\square}=T_{m \unlhd}-T_{e \rrbracket}
$$

Where $M$ is the moment of inertia.

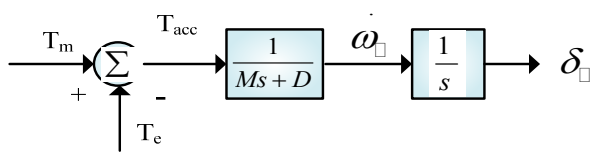

Fig. 3. Block diagram of generator rotor angle.

\subsection{Model Reduction and Tuning of Kp and Ki}

As was mentioned in section II, the tuning of $K_{p}$ and $K_{i}$ is very important to the trade-off between the AGC tracking performance and the governor performance. Fig. 4 shows the block diagram of generator unit with governor model. This block diagram is the transfer function $\mathrm{G}(\mathrm{s})$ in Fig. 1.

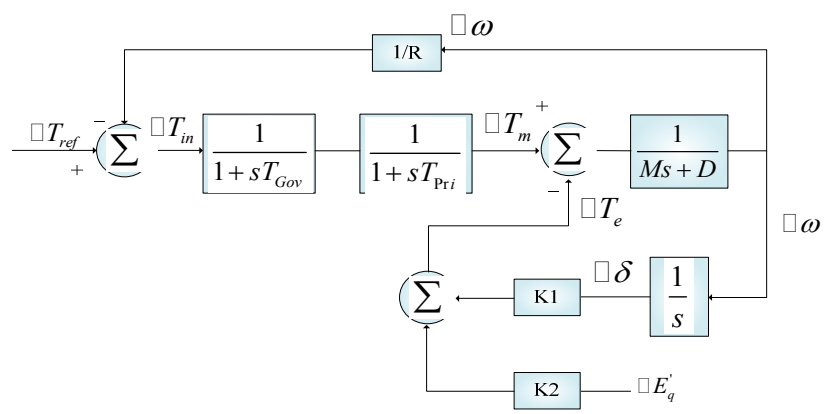

Fig. 4. Block diagram of generating unit. 
The parameters and initial conditions of the simplified system are shown in Table 2.

Table 2. Initial conditions and parameters of each generator

\begin{tabular}{|c|c|c|c|c|c|c|c|c|c|}
\hline No & \multicolumn{2}{|c|}{$X_{d}$} & \multicolumn{2}{|c|}{$X_{e}$} & \multicolumn{2}{|c|}{$P$} & \multicolumn{2}{|c|}{$E_{q}$} & $\delta$ \\
\hline Gen 1 & \multicolumn{2}{|c|}{0.0358} & \multicolumn{2}{|c|}{0.001} & \multicolumn{2}{|c|}{4.5} & \multicolumn{2}{|c|}{1.1018} & 8.37 \\
\hline \multirow[t]{2}{*}{ Gen2 } & \multicolumn{2}{|c|}{1.79} & \multicolumn{2}{|c|}{0.001} & \multicolumn{2}{|c|}{0.5} & \multicolumn{2}{|c|}{1.3424} & 41. \\
\hline & \multicolumn{2}{|c|}{$D$} & \multicolumn{2}{|c|}{$M$} & \multicolumn{2}{|c|}{$T_{g o v}$} & \multicolumn{2}{|c|}{$T_{p r i}$} & \\
\hline Gen 1 & \multicolumn{2}{|c|}{2} & \multicolumn{2}{|c|}{21.552} & \multicolumn{2}{|c|}{0.21} & \multicolumn{2}{|c|}{0.52} & \\
\hline Gen2 & \multicolumn{2}{|c|}{2} & \multicolumn{2}{|c|}{2.694} & & & \multicolumn{2}{|c|}{0.52} & \\
\hline \multicolumn{2}{|c|}{ No } & \multicolumn{2}{|c|}{$K_{1}$} & \multicolumn{2}{|c|}{$K_{2}$} & & & & $K_{4}$ \\
\hline \multicolumn{2}{|c|}{ Gen 1} & \multicolumn{2}{|c|}{10.99} & \multicolumn{2}{|c|}{1.071} & & & \multicolumn{2}{|c|}{-0.2298} \\
\hline \multicolumn{2}{|c|}{ Gen2 } & \multicolumn{2}{|c|}{1.8132} & \multicolumn{2}{|c|}{2.240} & & & \multicolumn{2}{|c|}{3.4704} \\
\hline
\end{tabular}

Gen 2 is the generator whose GUC is to be tuned, while Gen 1 is the one which is equivalent to the rest of the generators in the system. The transfer function of Gen 2 is given by equation (16).

$G(s)=$

$\frac{6.28 s^{2}+6.14 s^{1}+1.09}{1.02 s^{6}+4.84 s^{5}+19.50 s^{4}+32.48 s^{3}+30.51 s^{2}+10.94 s^{1}+1.09}$

We obtained linearized minimum phase sixth-order model of Gen 2 including governor. The model need to be reduced in order to calculate the proper gains of GUC for the tuning of the controller based on the system model.

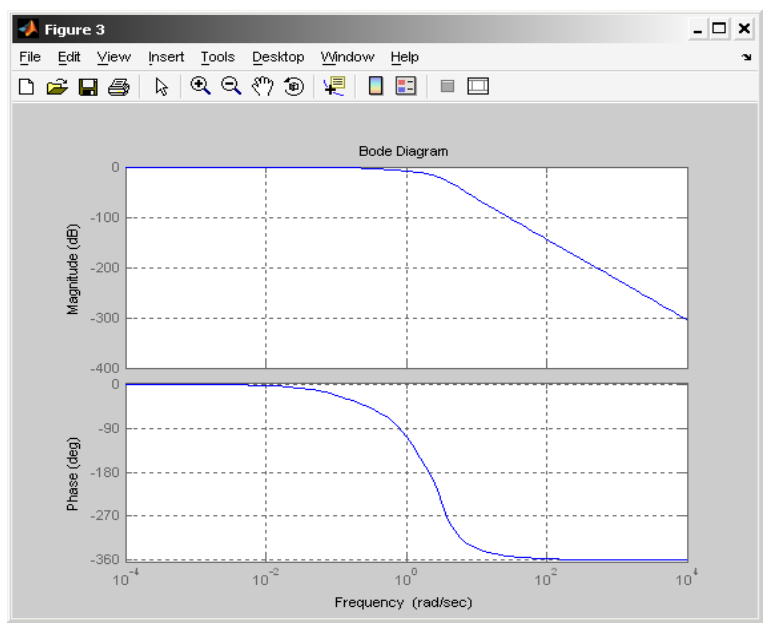

Fig. 5. Bode plot of the Gen 2 system.

It is important to give more weights to the low frequency ranges in the model reduction in order to yield satisfactory results. This allows the reduced model to have modeling error at the high frequency range, as modeling error at the low frequency range deteriorates the performance of the control systems.

\section{Model Reduction to the First-order System}

The first-order reduced system can be obtained from equation (17).

$$
\begin{gathered}
\|G(j w)\|=\left\|G_{r e d}(j w)\right\| \text { at } w=0, \\
\|G(j w)\|_{d b}=\left\|G_{r e d}(j w)\right\|_{d b}=-3 \mathrm{db} \text { at } w=w_{n} \\
G_{r e d}(j w)=\frac{K}{1+j w / w_{n}}
\end{gathered}
$$

We can leave the modeling error to high frequency ranges by making the magnitude of the two systems the same at the two low frequency points $-w=0$, and $w=w_{n}$.

The following can be obtained:

$$
\begin{array}{ll}
k=1 & \text { from }\|\mathrm{G}(0)\|=\left\|\mathrm{G}_{\text {red }}(0)\right\|=1, \\
w_{n}=0.25 & \text { from }\left\|\mathrm{G}\left(j w_{n}\right)\right\|_{d b}=\left\|G_{r e d}\left(j w_{n}\right)\right\|_{d b}=-3 \mathrm{db}
\end{array}
$$

The reduced system is given by equation (19).

$$
G_{r e d}(s)=\frac{1}{1+4 s}
$$

The bode magnitude plot of the original system and the first-order reduced system is shown in Fig. 6.

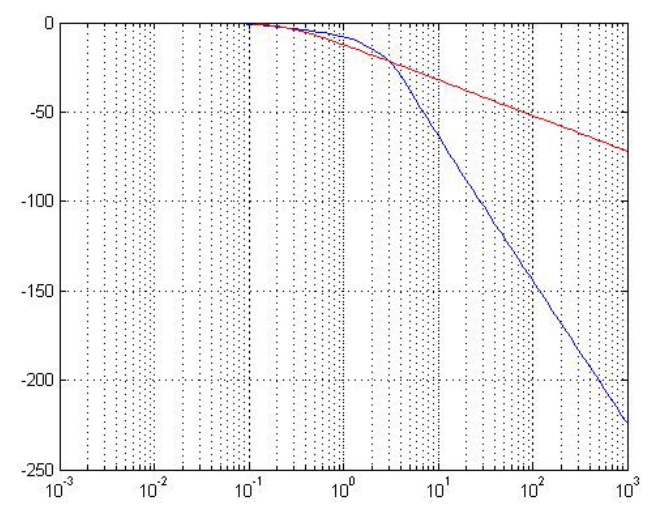

Fig. 6. Bode magnitude plot of the first-order reduced system $1 /(1+4 s)$ and original Gen 2 system.

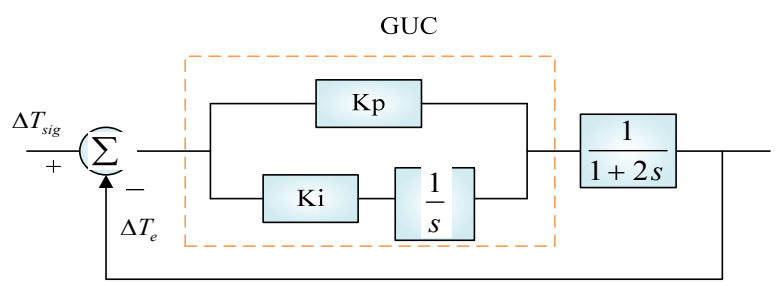

Fig. 7. Simplified block diagram of the first-order reduced generating unit.

Equation (20) is the transfer function of the closed loop system shown in Fig. 7.

$$
\frac{\Delta T_{e}}{\Delta T_{\text {sig }}}=\frac{K_{p} s+K_{i}}{4 s^{2}+\left(1+K_{p}\right) s+K_{i}}
$$

Equation (20) can be rearranged to be equation (21) by 
letting $K_{p}=4 K_{i}$.

$$
\frac{\Delta T_{e}}{\Delta T_{\text {sig }}}=\frac{K_{p} s+K_{i}}{\left(K_{p} s+K_{i}\right)\left(1+\frac{4}{K_{p}} s\right)}=\frac{1}{1+\frac{4}{K_{p}} s}
$$

The response of GUC can then have the form of firstorder exponential function.

$$
\begin{gathered}
T_{C L}=\frac{1}{K_{i}} \quad K_{p}=4 K_{i} \\
\Delta T_{\text {sig }}-\frac{1}{1+T_{C L} S} \Delta T_{e}
\end{gathered}
$$

Fig. 8. Closed loop transfer function obtained by letting

$$
K_{p}=4 K_{i} \text {. }
$$

\subsection{Simulation and Test Results}

As the typical response time of the governor ranges from 6 to 10 seconds, the GUC response time constant is expected to be 1 to couple of minutes. We compared the GUC performance between the cases when the closed loop time constant is 60 and 120 seconds. We set the closed loop time constants to 60 and 120 seconds by deciding $\mathrm{Ki}=1 / 60, \mathrm{Kp}$ $=1 / 15$ and $\mathrm{Ki}=1 / 120, \mathrm{kp}=1 / 30$, respectively.

For the simulation, we assumed that only one generator is involved in LFC control, while the remaining generators do not participate in the LFC control. Fig. 9 shows the AGC target signal generated by the sum of ED and LFC and the generator outputs with GUC on and off. Figs. 9(a) and 9(b) are for the case with two closed loop time constants, 60 and 120 seconds, respectively. The AGC target and power output were initially set to 0.5 and the load was increased by $0.05 \mathrm{pu}$. When GUC is set to off state (dashed line), the governor action raised the power output but there remained a steady-state deviation between the AGC target and the power output. The power output with GUC set to on state (dashed dot line) initially decreased and then increased until it reached the AGC target.

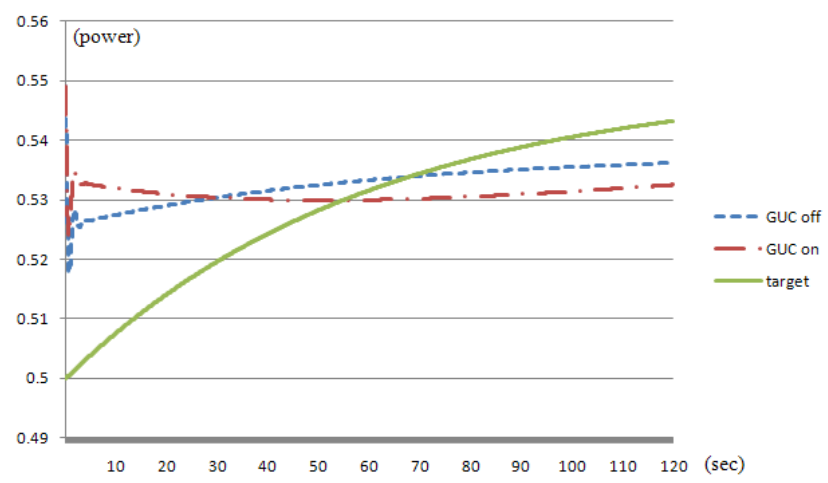

(a) $\mathrm{Kp}=1 / 15 ; \mathrm{Ki}=1 / 60$

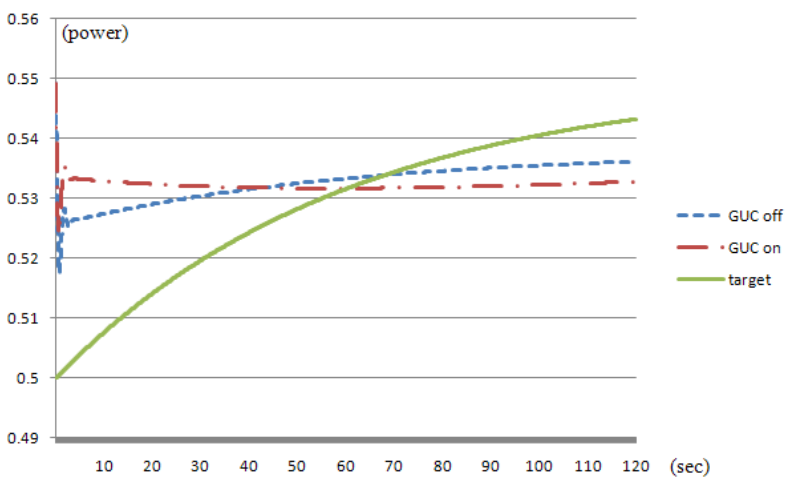

(b) $\mathrm{Kp}=1 / 30, \mathrm{Ki}=1 / 120$

Fig. 9. Power output with GUC on and off.

Fig. 10 shows the test result applied to the real power system. The generator output follows the random AGC target signal well.

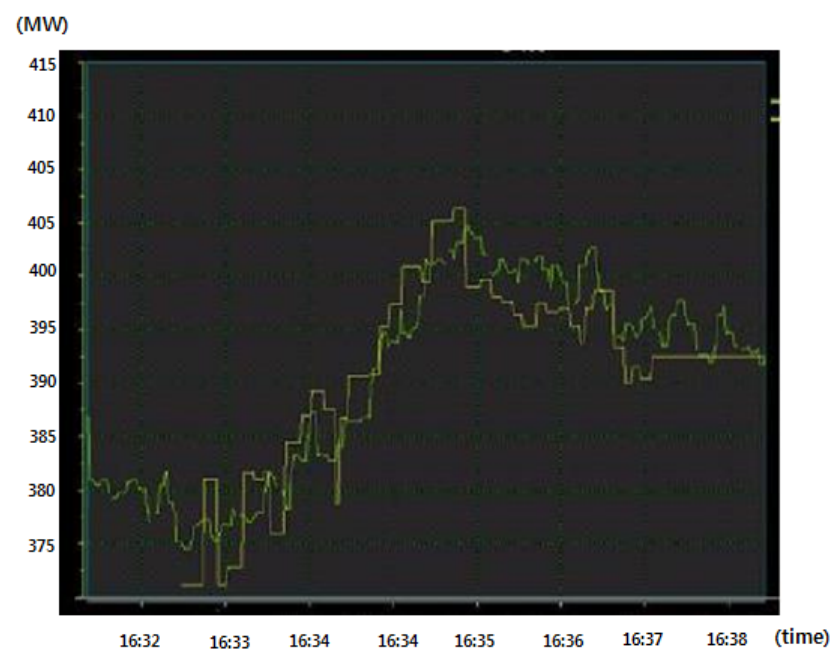

Fig. 10. AGC target (test signal) and generator output.

\section{Conclusion}

AGC in K-EMS consists of ACE, TED, and GUC control blocks. The tuning of ACE and TED control blocks can be accomplished by setting time constants. The time constants should be large enough not to conflict with governor actions. As GUC is a tracking control block, closed loop controller and tuning should be included in order to obtain proper performance. Well-tuned GUC guarantees that the generator power output will follow AGC target while not deteriorating governor performance.

\section{Acknowledgments}

This work was supported by the 2008 Hongik University Research Fund. 


\section{References}

[1] N. Jaleeli, Donald N. Ewart, et al, "Understanding Automatic Generation Control," Transactions on Power Systems, Vol 7, No. 3, pp 1106-1122, August 1992.

[2] "IEEE Recommended Definitions of Terms for Automatic Generation Control on Electric Power Systems", IEEE Std 94-1991 pp 5.

[3] S. Velusami, S. Ganapathy, "Decentralized LoadFrequency Control of Interconnected Power Systems with SMES Units and Governor Dead Band using Multi-Objective Evolutionary Algorithm," Journal of Electrical Engineering \& Technology Vol. 4, No. 4, pp. 443-450 December 2009.

[4] Z. Y. Dong, J. J. Ford, G. Ledwich, H. Bevrani "On Power System Frequency Control in Emergency Conditions," Journal of Electrical Engineering \& Technology, Vol. 3, No. 4, pp. 499-508, december. 2008.

[5] Jian Wu, Yong Cheng, Schulz, N.N., "Overview of Real-Time Database Management System Design for Power System SCADA System", SoutheastCon, 2006. Proceedings of the IEEE, pp 62-66, April 2006.

[6] Charles L. Phillips, H. Troy Nagle, Digital Control System Analysis and Design, Prentie-Hall, Inc 1984 p.225-226.

[7] John J. Grainger, William D. Stevenson, Jr., Power System Analysis, McGraw-Hill, Inc. 1994.m.

[8] "IEEE Recommended Definitions of Terms for Automatic Generation Control on Electric Power Systems", IEEE Std 94-1991 pp 7-10.

[9] Korean Energy Management System Technical Specification, KPX, 2006.
[10] Norman S. Nise, Control Systems Engineering, 4th ed., A John Wiley \& Sons, pp. 503-546.

[11] P.M.Anderson \& A.A Fouad. Power System Control and Stability, 2nd ed., A John Wiley \& Sons, pp. 14, 99, 132, 222-225.

[12] Norman S. Nise, Control System Engineering, $4^{\text {th }}$ ed., A John Wiley \& Sons, pp. 188-190.

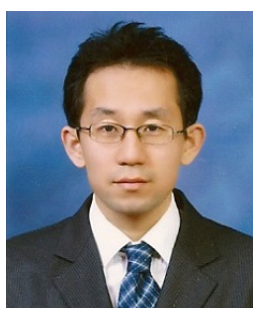

Min-Su Park received his B.S. and M.S. degrees from Hongik University, Korea in 2006 and 2008, respectively, and is currently a Ph.D. candidate student in the same university. His research interests are power system control and stability.

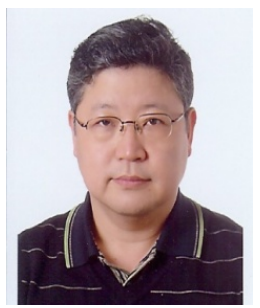

Yeong-Han Chun received his B.S. and M.S. degrees from Seoul National University, Korea in 1983 and 1985, respectively, and his Ph.D. degree from Tokyo University, Japan in 1997. He was with the Korea Electro-technology Research Institute (KERI) from 1985 to 2002 and, joined Hongik University where he is currently an Associate Professor of Electrical Engineering. His research interests are power system control and stability. 